\title{
A verocytotoxin-producing $E$. coli outbreak with a surprisingly high risk of haemolytic uraemic syndrome, Denmark, September-October 2012
}

B Soborg (bot@ssi.dk), , , S G Lassen ${ }^{1}$, L Müller ${ }^{1}$, T Jensen ${ }^{3}$, S Ethelberg ${ }^{1,4}$, K Mølbak ${ }^{1}$, F Scheutz ${ }^{4}$

1. Department of Infectious Disease Epidemiology, Statens Serum Institut, Copenhagen, Denmark

2. European Programme for Intervention Epidemiology Training (EPIET), European Centre for Disease Prevention and Control, $(E C D C)$, Stockholm, Sweden

3. The Danish Veterinary and Food Administration, Copenhagen, Denmark

4. Department of Microbiology and Infection Control, Statens Serum Institut, Copenhagen, Denmark

Soborg B, Lassen SG, Müller L, Jensen T, Ethelberg S, Mølbak K, Scheutz F. A verocytotoxin-producing E. coli outbreak with a surprisingly high risk of haemolytic uraemic syndrome, Denmark, September-October 2012. Euro Surveill. 2013;18(2):pii=20350. Available online: http://www.eurosurveillance.org/ViewArticle. aspx?Articleld $=20350$

Denmark faced an outbreak of verocytotoxin-producing $E$. coli (VTEC) 0157:H7 infections in autumn 2012. Thirteen cases were diagnosed of which eight had haemolytic uraemic syndrome (HUS). Epidemiological investigations suggested ground beef as the vehicle of the outbreak. The outbreak strain had a rare toxin gene subtype profile: eae, vtx1a and vtx2a, and a high proportion of HUS (62\%) among cases, a finding previously linked with the outbreak subtype profile. Toxin subtyping can be useful to identify high risk VTEC strains.

In late September 2012, a paediatric department in a Central Copenhagen Hospital notified Statens Serum Institut (SSI) of four cases of haemolytic uraemic syndrome (HUS). This led to the initiation of an outbreak investigation.

HUS and infections with verocytotoxin-producing E. coli (VTEC) are individually notifiable in Denmark. Furthermore, VTEC infections are laboratory-notifiable. Around 180 cases are reported each year, of which serogroup 0157 constitute 15-20\% [1]. Previously, Denmark has experienced only two general outbreaks of VTEC infection. In 2006 an outbreak of VTEC 0157 affected 25 cases, with organic pasteurised milk as the source [2]. In 2007, an outbreak of VTEC 026 affected 20 cases, with an organic fermented beef sausage as the source [3]. In addition, Denmark reported 26 cases during the large VTEC 0104 outbreak in Germany in 2011 [4]. The annual number of HUS cases in Denmark ranges from two to six [1]. Subtyping of verocytotoxin genes are done at $\mathrm{SSI}$ as described in [5].

\section{Outbreak investigation}

For this outbreak, we defined a confirmed case as an individual with a laboratory-confirmed infection with VTEC 0157 eae, vtx1a and vtx2a diagnosed after 1 August 2012, or a person diagnosed with HUS in the same time period and a serology-confirmed VTEC
O157 infection. A probable case was a person without laboratory-confirmed VTEC infection, diagnosed with HUS after 1 August 2012, or a person diagnosed with VTEC 0157 infection in the same period, but without subtyping, or a person diagnosed with VTEC eae, vtxia and $v$ txza infection in the same period, but without 0 -typing. The date 1 August was chosen in order to identify any early cases.

On 22 October, after laboratory confirmation of VTEC 0157 in two patients, SSI notified Danish clinical laboratories of a possible VTEC outbreak to increase case ascertainment and reporting. We interviewed patients (or their parents for children under 15 years of age) by telephone, using a structured trawling questionnaire. Early investigations had pointed to ground beef as the possible outbreak source, and information on purchase dates and other possible labelling information of ground beef was collected and presented to the supermarkets for further tracing of possible batch identification. The Danish Veterinary and Food Administration traced back the ground beef.

SSI performed live-slide agglutination on the submitted isolates upon arrival, and all VTEC 0157-positive isolates were typed immediately with the PCR protocol for subtyping of $v t x$ genes [5]. Further testing included conventional (and confirmatory) O:H serotyping, Vero cell assay, fermentation of sorbitol, production of betaglucuronidase, presence of additional virulence genes by dot blot hybridisation and/or PCR, and pulsed-field gel electrophoresis (PFGE). We asked patients who were culture-negative for VTEC $0_{157}$ to submit a serum specimen for serology testing for antibodies against VTEC 0157.

\section{Findings}

We identified 13 cases of VTEC 0157 (11 confirmed and two probable). Eight cases had HUS. The cases belonged to nine families. Eleven reported symptoms, 
Probable and confirmed cases of symptomatic verocytotoxin-producing E. coli O157 by date of onset, Denmark, September -October $2012(n=11)$

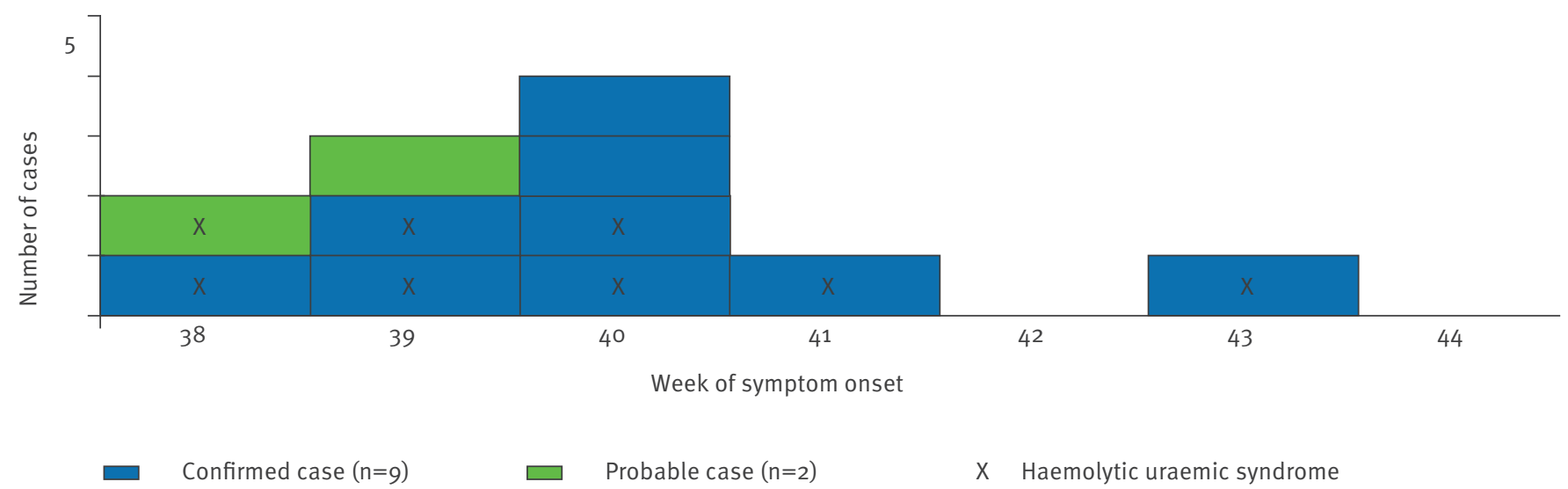

The two asymptomatic cases found by screening are not depicted in the figure.

whereas two were diagnosed when we screened healthy family members. Date of disease onset ranged from 18 September to 28 October 2012 (Figure). The cases were distributed throughout the country, eight were female, and the median age was 14 years (range: 3-68 years).

Hypothesis-generating interviews suggested that the source of the outbreak was food served in the households. Most affected families had young children, ate traditional (non-vegetarian) Danish food and lived in provincial towns. The most frequent food exposure reported was ground beef: all cases had eaten ground beef during the incubation period, six in the form of pan-fried ground beef ("hakkebøffer"). Two households mentioned the beef still being red in the centre when eaten. No other food items were common to all cases. The families had purchased food in a variety of different supermarket chains. Trace-back of the beef indicated that it was of Danish origin and narrowed the possibilities down to two major Danish slaughterhouses and to two consecutive slaughter days. However, identification of a single food producer was impossible and no further action was taken.

We compared interview information from the current outbreak with that of a salmonella outbreak occurring in 2011 in the same months (to account for possible changes in eating habits with the seasons) [6], where a comparable hypothesis-generating questionnaire was used. The nine families involved in the 2012 VTEC outbreak reported similar frequencies of consumption as the 2011 salmonella cases for almost all food items. However, all nine case-families in the 2012 VTEC outbreak reported eating ground beef, compared with 14 of 24 case-families in the salmonella outbreak $(p=0.03$; Fisher's exact-test).

The outbreak strain was a typical VTEC 0157: $\mathrm{H}_{7}$, eae positive, non-sorbitol fermenting, negative for betaglucuronidase. However, it had a rare toxin subtype profile including the genes vtx1a and vtx2a. All strains had the same PFGE profile, which had not been reported previously among Danish VTEC isolates. The Table shows the distribution of the total number of VTEC 0157 cases diagnosed and sub-typed in Denmark from 1997 to 2012, stratified by toxin profile. For each toxin profile, the proportion of cases with HUS is shown. VTEC infections containing the vtxza toxin profile were associated with a higher number of HUS cases (from $3 \%$ to above $20 \%$ ), and VTEC infections containing the $e a e+v t \times 1 a+v t \times 2 a$ profile were associated with even higher frequencies of HUS cases (33\%).

\section{Discussion}

The present outbreak represents the first food-borne outbreak of a highly virulent VTEC 0157 in Denmark. Subtyping of the toxin genes allowed rapid identification and classification of cases. In addition, toxin subtyping was of relevance for the overall risk assessment. The combination of $v t \times 1 a$ and $v t \times 2 a$ in the toxin profile of the outbreak strain is rare and appears to be associated with a high risk of progression to HUS as also indicated by previously subtyped VTEC 0157 strains. This emphasises that within the VTEC 0157 group, there is a diversity of strains with different potential of causing HUS.

As a surrogate for an analytical epidemiological investigation, we compared case exposure to ground beef 
Verocytotoxin-producing E. coli O157 cases and proportion of haemolytic uraemic syndrome by toxin profile, Denmark, 1 January 1997-31 July $2012(n=212)$

\begin{tabular}{|l|c|c|c|}
\hline Toxin profile & $\begin{array}{c}\text { Total number } \\
\text { of cases }\end{array}$ & $\begin{array}{c}\text { Number of } \\
\text { HUS cases }\end{array}$ & $\begin{array}{c}\text { Proportion } \\
\text { of HUS }\end{array}$ \\
\hline$e a e+v t \times 1 a$ & 8 & 0 & 0 \\
\hline$e a e+v t \times 1 a+v t \times 2 c$ & 85 & 0 & 0 \\
\hline$e a e+v t \times 2 c$ & 31 & 1 & $3 \%$ \\
\hline$e a e+v t \times 2 a+v t \times 2 c$ & 45 & 11 & $24 \%$ \\
\hline$e a e+v t \times 2 a$ & 37 & 11 & $30 \%$ \\
\hline$e a e+v t \times 1 a+v t \times 2 a$ & 6 & 2 & $33 \%$ \\
\hline
\end{tabular}

HUS: haemolytic uraemic syndrome.

with similar exposure in a recent salmonella outbreak. This case $\neg$-case comparison suggested that more families than expected had consumed ground beef in the present outbreak. In addition, these families had prepared it in a way that allowed for part of the beef to remain uncooked. This suggests that this outbreak was caused by ground beef, a common source of VTEC outbreaks. Ground beef has a short shelf life (seven days), which may have limited the size of this outbreak. If the vehicle of the outbreak had been a food item with longer shelf life, the public health impact of this high risk HUS-associated VTEC outbreak could have been much larger.

Based on the findings of this outbreak we suggest performing toxin subtyping in similar outbreak situations in order to quickly identify high risk VTEC strains and thereby aid risk assessment of the outbreak.

\section{References}

1. VTEC, laboratorieanmeldelsespligtige sygdomme. [VTEC, laboratory-notifiable diseases]. Copenhagen: Statens Serum Institut. [Accessed December 2012]. Danish. Available from: http://www.ssi.dk/Smitteberedskab/Sygdomsovervaagning/ Sygdomsdata.aspx?sygdomskode $=$ STEC $\&$ xaxis $=$ Aar $\&$ show $=\& d$ atatype $=$ Laboratory\&extendedfilters $=$ False\#HeaderText

2. Jensen C, Ethelberg S, Gervelmeyer A, Nielsen EM, Olsen $\mathrm{KE}$, Molbak K. First general outbreak of Verocytotoxinproducing Escherichia coli $0_{157}$ in Denmark. Euro Surveill. 2006;11(2):pii=597. Available from: http://www. eurosurveillance.org/ViewArticle.aspx?Articleld=597

3. Ethelberg S, Smith B, Torpdahl M, Lisby M, Boel J, Jensen T, et al. Outbreak of non-0157 Shiga toxin-producing Escherichia coli infection from consumption of beef sausage. Clin Infect Dis. 2009;48(8):e78-e81.

4. Müller L, Ethelberg S, Kjelsø C, Mølbak K, Scheutz F, et al. VTEC 0104 outbreak in Germany. EPI-NEWS. 2011;27-33. Available from: http://www.ssi.dk/English/News/EPINEWS $/ 2011 / \mathrm{No}_{2} 2027-33 \% 20-\% 202011$.aspx

5. Scheutz F, Teel LD, Beutin L, Pierard D, Buvens G, Karch H, et al. Multicenter evaluation of a sequence-based protocol for subtyping shiga toxins and standardizing Stx nomenclature. Clin Microbiol. 2012;50(9):2951-63.

6. Müller L. Copenhagen: Statens Serum Institut. Outbreak of salmonella Strathcona. 2012. EPI-NEWS. 2012;4. Available from: http://www.ssi.dk/English/News/EPI-NEWS/2012/ No\%204\%20-\%202012.aspx 